\title{
Microwave-Assisted Polyesterification Process in Bulk and Aqueous Media
}

\author{
Nassima Mazouzi-Sennour*, François Henry \\ Equipe "Systèmes Polymères Complexes", Institut de Chimie et des Matériaux Paris-Est, UMR 7182 \\ CNRS-Université Paris-Est Créteil, Thiais, France \\ Email: "nassima mazouzi@yahoo.fr
}

Received 7 December 2013; revised 16 January 2014; accepted 23 January 2014

Copyright $@ 2014$ by authors and Scientific Research Publishing Inc.

This work is licensed under the Creative Commons Attribution International License (CC BY).

http://creativecommons.org/licenses/by/4.0/

(c) (i) 0pen Access

\begin{abstract}
Microwave irradiation in polymer chemistry is an emerging research field. This type of heating can enhance the rate of reaction and improve the specific characteristics of the formed polymer. In this context, the present paper focuses on selective microwave (MW) heating and its influence on the polyesterification reaction. As a reaction model, the polyesterification of sebacic acid with decanediol, in bulk and in aqueous emulsion is investigated. The reaction was catalyzed by using 4-dodecylbenzenesulfonic acid (DBSA), which plays a catalytic and surfactant role. Both in bulk and in aqueous media, a polyester with higher molecular weight is obtained in MW heating compared to the conventional heating.
\end{abstract}

\section{Keywords}

\section{Microwave; Polyesterification; Emulsion; 4-Dodecylbenzenesulfonic Acid}

\section{Introduction}

Usually, polymer latexes are obtained by emulsion polymerization, but this technique is limited to free radical polymerization. Using other techniques such as suspension, precipitation or interfacial, polycondensation of a wide range of polymers have been synthesized: polycarbonates [1], polyesters and polyamides [2], [3].

The reversible polycondensation involving the elimination of water molecule is usually conducted in bulk at relatively high temperature $\left(180^{\circ} \mathrm{C}-230^{\circ} \mathrm{C}\right)$ in order to remove water from the system. This allows the shift of the equilibrium to form high molecular weight polymers with high yield reaction [4]. In an apparent contradiction to this process, the polycondensation of silanol emulsified in water in the presence of stabilizers as sulfonic acid allowed the formation of polydimethylsiloxane oligomers [5]. These results were then transposed to the poly-

*Corresponding author. 
condensation of diacid, diol and aliphatic aldehydes dispersed in water [6], [7]. These systems do not need to remove the water.

Recently, a few research groups were interested in the synthesis of polyesters or esters in the presence of a large amount of water. Saam et al. studied the polycondensation of hydrophobic diacids and diols in suspension in water using different sulfonate surfactants [6], [8]. They showed that low molecular weight polyesters may be obtained in aqueous media. The main advantage of this process is the transfer of the water formed in the hydrophobic organic phase to the aqueous phase. A kinetic study of the reaction allowed them to conclude that the hydrolysis takes place in the organic phase but this phenomenon is limited by the insolubility of water in the hydrophobic phase [9], [10].

The polyesterification emulsion in water was observed in hydrophobic monomers. The principle of the polyesters synthesis in aqueous media is the creation of mini-reactors whose hydrophobic heart containing monomers constitutes the reaction site. These mini-reactors are supported and protected by a surfactant, which is adsorbed to their surfaces (Figure 1).

Manabe et al. studied the esterification of hydrophobic monofunctional compounds in aqueous suspension [11], [12]. They investigated the kinetics of esterification with different acid catalysts and showed that the most hydrophobic of them is also the most effective because it can be adsorbed on the particle surface and catalyze the reaction at the interface. They use surfactant which also plays a catalytic role as dodecylbenzenesulfonic acid (DBSA), called CATASURF.

More recently, Barrere et al. were interested in the synthesis of polyester in miniemulsion in order to obtain stable polymer latex [13]. They concluded that the monomers hydrophobicity plays an important role in the development of the polyesterification. This study also showed that the change of the droplets environment, as the ionic strength or the interface nature, has no influence on the equilibrium of the reaction. Additionally, low molecular weight polyesters were obtained. They were mainly used as a precursor for the hybrid polyester/polystyrene polymers. Tanaka et al. studied the polyesterification of sebacic acid and decanediol in aqueous emulsion [14]. A polyester with $5000 \mathrm{~g} / \mathrm{mol}$ molecular weight was obtained. Takasu et al. also studied the polyesterfication in aqueous emulsion by using scandium trisdodesyl sulfate $\left[\mathrm{Sc}(\mathrm{DS})_{3}\right]$, which is a combination between scandium chloride (III) used as a Lewis acid and sodium dodecyl sulfate (SDS) used as a surfactant [15]. This type of surfactant-combined catalyst was already used for various organic syntheses in aqueous media [16]. However, the molecular weight of the obtained polyester was comparable to that of the polyesters obtained using the DBSA.

The use of enzymatic catalysis was also investigated. Kobayashi et al. studied the polyesterification of sebacic acid and octanediol in aqueous media using the enzyme Pseudomonas cepacia lipase as catalyst [17]-[19]. A polyester with $1600 \mathrm{~g} / \mathrm{mol}$ molecular weight was obtained.

In recent years, a lot of studies were devoted to the application of microwave (MW) heating in polymer synthesis [20]. This type of heating is more penetrative than the conventional one [21]. It offers a clean, convenient and inexpensive method of heating which often results in higher yields and shorter reaction times [22]. The polyesterification emulsion in water has recently been described. However, low molecular weights (1000 g/mol) were obtained [23].

In this context, the aim of the present study is to investigate the polyesterification in emulsion and in bulk. We principally focus on selective MW heating and its influence on the polyesterification process.

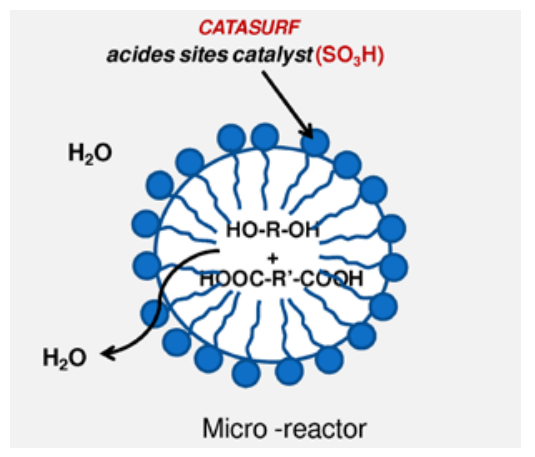

Figure 1. Principle of the ester synthesis in aqueous medium. 


\section{Experimental Details}

\subsection{Chemicals Reagents}

Sebacic acid (99\%), decanediol (98\%), hexadecane and 4-dodecylbenzenesulfonic acid (DBSA, mixture of isomers 95\%) were purchased from Sigma Aldrich Chemicals.

\subsection{Materials}

Reactions were performed in two types of MW applicators:

Pseudo-single-mode applicator: the electromagnetic wave exiting the waveguide and arriving on the sample holder was slightly depolarized. With this type of MW applicator, the power to be applied can be set manually using a calibrated potentiometer.

Multimode applicator: it is a commercial MW (Synthos 3000, Anton-Paar) equipped with two magnetrons able to provide $1400 \mathrm{~W}$ of continuous MW power at $2.45 \mathrm{GHz}$. The control system supplied with the instrument allows to work with either controlled temperature or irradiation power.

\subsection{Polymer Synthesis}

The polyesterification of sebacic acid with decanediol was synthesized according to Scheme 1.

\subsubsection{Microwave-Assisted Bulk Polyesterification}

As a classical recipe, 0.01 mole of sebacic acid, 0.01 mole of decanediol and $0.577 \times 10^{-3}$ mole of 4-dodecylbenzenesulfonic acid (DBSA) was melted at temperature of $120^{\circ} \mathrm{C}$ under conventional heating, and then the reaction was continued under microwave irradiation at the same temperature. In the case of the reaction without catalyst, the polyester was synthesized following the same procedure but without using the catalyst. In these experiments, a pseudo-single-mode applicator was used.

\subsubsection{Microwave-Assisted Emulsion Polycondensation}

In the first step, the emulsion was prepared under conventional heating. A 0.668 mole of Sebacic acid, 0.668 mole of decanediol and $4 \times 10^{-3}$ mole of hexadecane were melted at $120^{\circ} \mathrm{C}$, and then added at $90^{\circ} \mathrm{C}$ to $90 \mathrm{~g}$ of water containing $145 \mathrm{~g} / \mathrm{L}$ of 4-dodecylbenzenesulfonic acid. The mixture was sheared during $15 \mathrm{~min}$ by an Ultra $\operatorname{Turrax}^{\circledR}$ homogenizer. The hexacedan was used as a hydrophobic agent to slow down the Ostwald ripening phenomena [24] and to improve the droplets heart fluidity. In the present study, only the multimode applicator was used.

\subsection{Analysis}

\subsubsection{Size Exclusion Chromatography}

Average molar masses were determined by SEC using a Spectra Physics P100 pump with two PL gel-type columns connected in series (5 mm Mixed-C) from Polymer Laboratories. A Wyatt Technology Optilab rEX interferometric refractometer was used as the detector. $\mathrm{CHCl}_{3}$ was used as the eluent at a flow rate of $1 \mathrm{~mL} \cdot \mathrm{min}^{-1}$, and standards polystyrene were employed for calibration. The samples were dissolved at a concentration of 10 $\mathrm{mg} \cdot \mathrm{mL}^{-1}$ in $\mathrm{CHCl}_{3}$.

\subsubsection{Nuclear Magnetic Resonance Spectroscopy}

The liquid ${ }^{1} \mathrm{H}$ NMR spectra were recorded with a Brucker Avance II 400 at room temperature. $\mathrm{CDCl}_{3}$ was used as the solvent and the internal standard (7.24 ppm).

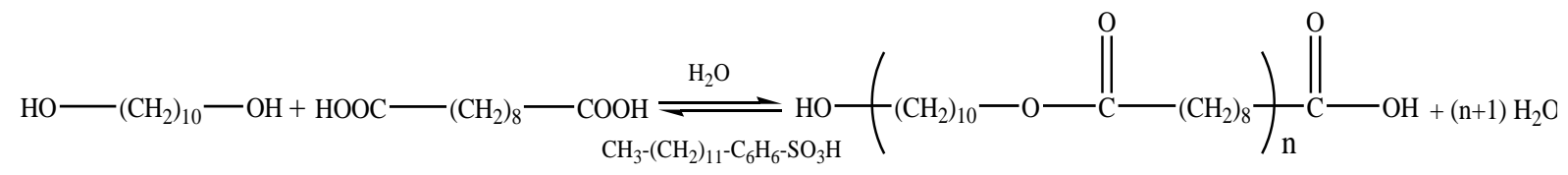




\subsubsection{Infrared Spectroscopy}

Attenuated total reflection Fourier transform infrared (ATR-FTIR) spectra were run with a Brucker Tensor 27 DTGS spectrophotometer. The spectra were recorded between 4000 and $450 \mathrm{~cm}^{-1}$ with an average of 30 consecutive scans with a resolution of $4 \mathrm{~cm}^{-1}$.

The polyesterification progress rate was determined by ATR-FTIR spectroscopy. The relative rate of the ester formation was calculated according to the following the formula:

$$
f_{\text {Esterification rate }}(\%)=\frac{A_{\text {ester }}}{A_{\text {ester }}+A_{\text {acid }}}
$$

This formula allows to plot the normalized curve of the ester formation where $A_{\text {ester }}$ corresponds to the integration of the $1730 \mathrm{~cm}^{-1}$ band resulting from the $\mathrm{C}=\mathrm{O}$ stretching vibration of ester group and $A_{\text {acid }}$ corresponds to the $1690 \mathrm{~cm}^{-1}$ band resulting from the $\mathrm{C}=\mathrm{O}$ stretching vibration of carboxylic acid group.

\section{Results and Discussion}

\subsection{Microwave-Assisted Bulk Polyesterification}

The polycondensation of sebacic acid and decanediol was performed under MW and conventional heating (CH). Table 1 and Figure 2 present a comparison between both types of heating in term of the obtained polyester molecular weight and in term of the polyesterification progress respectively. The comparison is made in both cases of the presence or not of a catalyst.

1) Without catalyst: the polyestrification rate under MW heating is higher than in the case of conventional heating, and it is increasingly significant as the applied power is important. However, the increase of the power induces a faster increase of the temperature which explains the acceleration of the polyesterification reaction.

On the other hand, after 30 min of reaction, a low molecular weight oligomers (DP $=3$ ) were obtained compared to the case with using a catalyst.

2) With catalyst: in this case, a significant progression of the polyesterification rate was observed. The application of two different power values did not have an effect on the polyestrification rate. However, MW heating enables the acceleration of the reaction by about $10 \%$ compared to conventional heating. Furthermore, MW activation allows obtaining of largest molecular weight polyester in a relatively short time compared to conventional heating.

\subsection{Microwave-Assisted Emulsion Polyesterification}

Table 2 summarizes the results of the polyesterification of decanediol and sebacic acid in water with the presence of DBSA. The reaction was conducted under MW heating in function of different parameters: temperatures, DBSA concentration and monomers dilution. One can note the increase of the polyester molecular weight with the increase of the DBSA concentration. A large amount of DBSA leads to an enhancement of its catalytic effect. This also improves the MW heating efficiency according to the increase of polarity. On the other hand, the polyester molecular weight was insensitive to the dilution of monomers. This is slightly surprising as water have a high dielectric constant.

The increasing of the water concentration in the dispersed phase would improve the microwaves absorption and convert them into energy. However, this was not observed in this study.

Table 3 shows comparison between pseudo-single-mode MW and conventional heating in the case of emulsion polyesterification of sebacic acid and decanediol in water with the presence of DBSA. The comparison is carried out for two different experimental procedures: in the first case, the monomers mixture was melted prior to emulsification (homogeneous condition) and in the second case all reagents were added to water at the same time (heterogeneous). In both cases higher molecular weight polyester was obtained under MW heating compared to the conventional one. These observations demonstrate the efficiency of MW activation, and in particular the pseudo-single-mode MW applicator. Indeed, in this type of applicator, the electromagnetic wave arriving on the sample is only slightly depolarized resulting in maximal absorption of the wave. The combination of adequate catalyst concentrations and water maximum absorption makes optimal the MW heating and improves the electromagnetic waves penetration. The latter are focused on the polar parts (ionizable dipole $\mathrm{SO}_{3}^{-}, \mathrm{H}^{+}$) of the catalyst and on the monomer polar functional groups increasing the collision frequency between these groups. 
Table 1. Comparison between microwave (MW) and conventional heating (CH) after 30 min of reaction.

\begin{tabular}{ccc}
\hline & & Type of heating \\
\cline { 2 - 3 } & MW & Conventional \\
\hline Applied Power $[\mathrm{W}]$ & 210 & - \\
Without catalyst $M_{w}(\mathrm{~g} / \mathrm{mole})$ & 755 & 975 \\
With catalyst $M_{w}(\mathrm{~g} /$ mole $)$ & 8668 & 5567 \\
\hline
\end{tabular}

Table 2. Polycondensation of sebacic acid and decanediol with DBSA in water under MW heating, a maximum generated power of $1200 \mathrm{~W}$ in the MW cavity.

\begin{tabular}{cccccc}
\hline [monomer] (mol/L) & {$[\mathrm{DBSA}](\mathrm{g} / \mathrm{L})$} & $\mathrm{T}\left({ }^{\circ} \mathrm{C}\right)$ & $\mathrm{t}(\mathrm{min})$ & $\mathrm{Mw}(\mathrm{g} / \mathrm{mol})$ & $\mathrm{Mw} / \mathrm{Mn}$ \\
\hline 0.2228 & 12 & 60 & 60 & 2924 & 1.9 \\
0.2228 & 12 & 70 & 60 & 2434 & 3.2 \\
0.668 & 36 & 90 & 60 & 2874 & 1.8 \\
0.2228 & 145 & 90 & 30 & 2892 & 2.2 \\
0.2228 & 145 & 70 & 60 & 3768 & 2.2 \\
0.668 & 145 & 90 & 60 & 2904 & 2.3 \\
0.668 & 145 & 70 & 60 & 3626 & 2.0 \\
\hline
\end{tabular}

Table 3. Comparison between MW and conventional heating.

\begin{tabular}{|c|c|c|c|c|c|c|}
\hline [monomer] (mol/L) & Heat & $\mathrm{T}\left(\mathrm{C}^{\circ}\right)$ & $\mathrm{t}(\mathrm{h})$ & $\mathrm{Mw}$ (g/mol) & $\mathrm{Mw} / \mathrm{Mn}$ & Yield (\%) \\
\hline 0.668 & MW a & 95 & 2 & 5900 & 1.8 & 50 \\
\hline 0.668 & $\mathrm{CH} b$ & 90 & 24 & 2837 & 1.9 & 52 \\
\hline 0.222 & MW c & 90 & 1 & 4031 & 1,8 & 30 \\
\hline 0.222 & $\mathrm{CH} \mathrm{d}$ & 90 & 24 & 2656 & 1,9 & 45 \\
\hline
\end{tabular}

${ }^{\mathrm{a}}[\mathrm{HD}]=1.3 \times 10^{-3}$ mole, $[\mathrm{DBSA}]=145 \mathrm{~g} / \mathrm{L}$ in $90 \mathrm{~g}$ of water. The reaction was carried out under microwave heating using pseudo single mode applicator. ${ }^{\mathrm{b}}[\mathrm{HD}]=1.3 \times 10^{-3}$ mole, [DBSA] $=145 \mathrm{~g} / \mathrm{L}$ in $90 \mathrm{~g}$ of water. The reaction was carried out under conventional heating. ${ }^{\mathrm{c}}[\mathrm{HD}]=0.43 \times 10^{-3}$ mole, $[\mathrm{DBSA}]=145 \mathrm{~g} / \mathrm{L}$ in $30 \mathrm{~g}$ of water. Heterogeneous media: all reagents were added to water at same time. The reaction was carried out under microwave heating using multiomode applicator. ${ }^{d}[\mathrm{HD}]=0.43 \times 10^{-3}$ mole, [DBSA] $=145 \mathrm{~g} / \mathrm{L}$ in 30 g of water. Heterogeneous media: all reagents were added to water at same time. The reaction was carried out under conventional heating

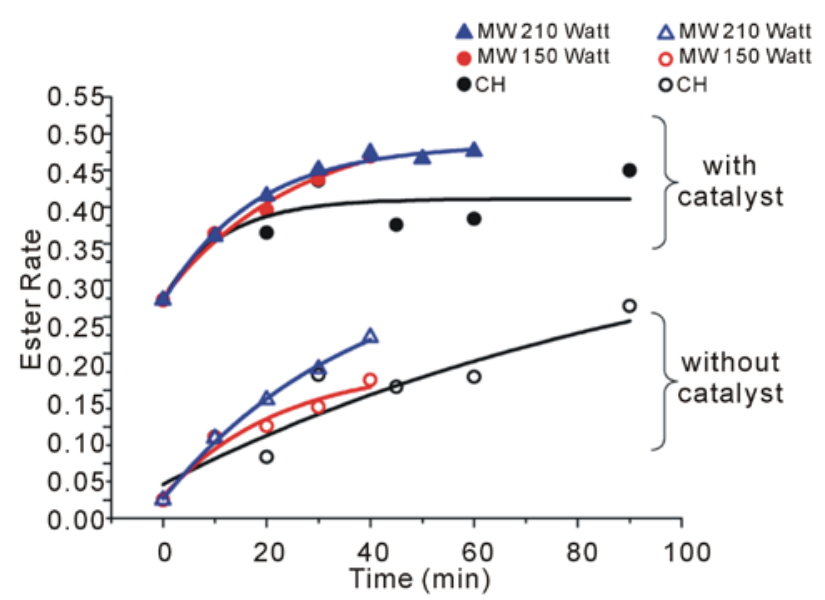

Figure 2. Polyesterification at $120^{\circ} \mathrm{C}$ in bulk under conventional and microwave heating.

Both effects can greatly accelerate the polycondensation reaction.

\subsection{Polymer Characterization}

Figure 3 illustrates the typical ATR-FTIR spectrum of a model polyester with the presence of a new band at 
$1731 \mathrm{~cm}^{-1}$ arising from the $\mathrm{C}=\mathrm{O}$ stretching vibration of ester groups and the absence of a detectable band at $1678 \mathrm{~cm}^{-1}$, from the $\mathrm{C}=\mathrm{O}$ stretching vibration of carboxylic acid groups.

${ }^{1}$ H-NMR spectroscopy also corroborated the formations of the expected polyesters (Figure 4). The spectrum presents proton signals at $\delta=3.59 \mathrm{ppm}$ (peak a) and $\delta=2.22 \mathrm{ppm}$ (peak c) which are characteristic of a polyester.

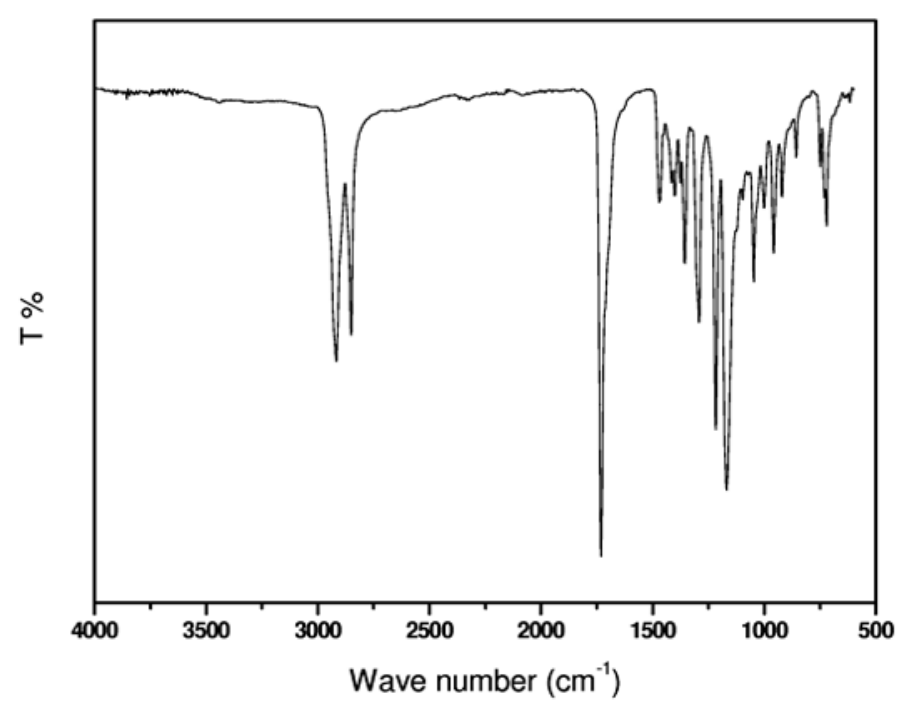

Figure 3. IR spectrum of polyester prepared from decanediol and sebacic acid.

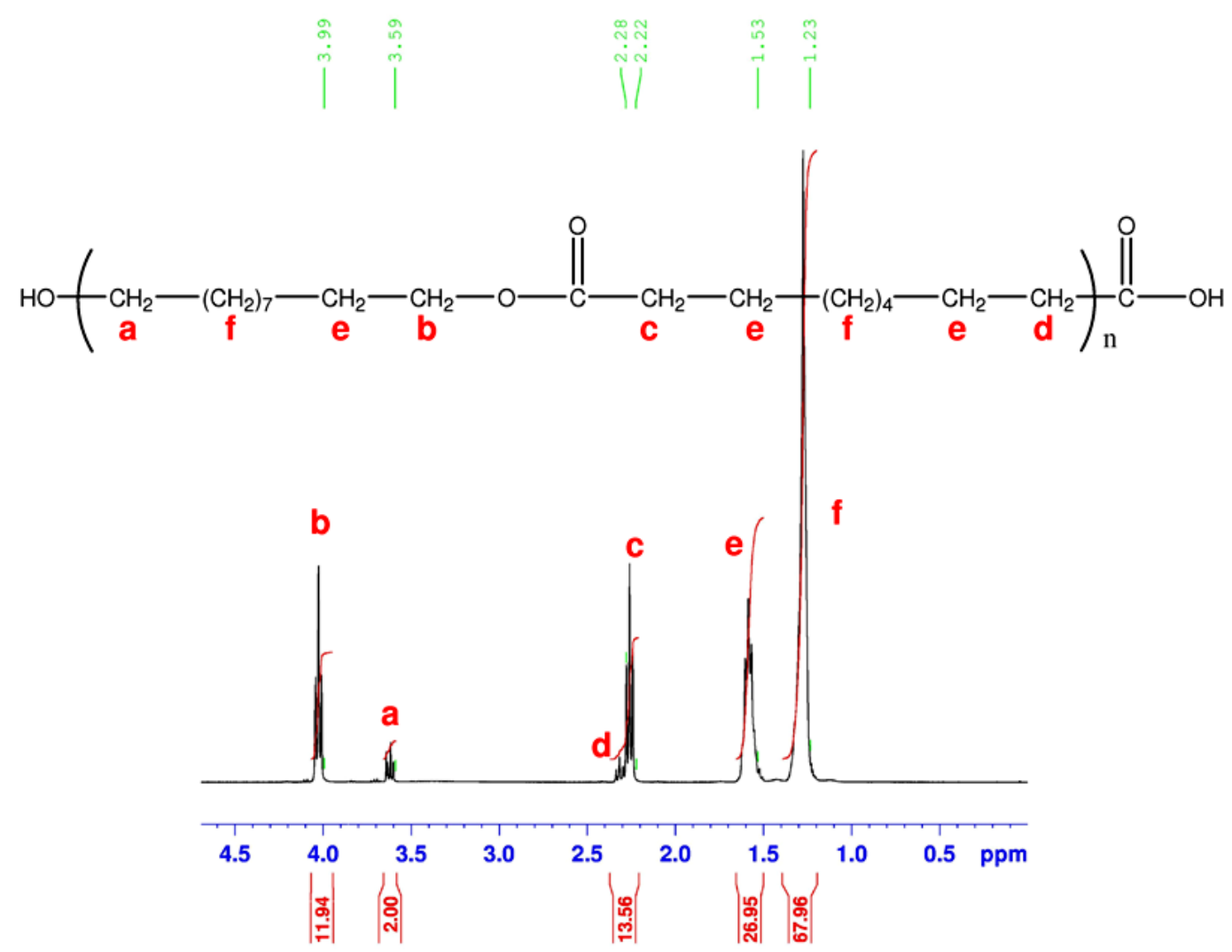

Figure 4. ${ }^{1} \mathrm{H}$ NMR spectrum of an aliphatic polyester prepared from decanediol and sebacic acid. 


\section{Conclusions}

Bulk polyesterification under microwave heating shows that the rate of polyestrification is more important compared to conventional heating. This was established either with or without a catalyst. In the case of the absence of a catalyst, lower molecular weights are obtained. However, with a catalyst, largest molecular weight polymers are obtained in a relatively short time compared to conventional heating.

In the case of emulsion polyesterification, the studied reactions consisted in mini-reactors (droplets) whose core is constituted of hydrophobic monomers with the presence of surfactant. The combination of water high dielectric loss $\left(\varepsilon^{\prime \prime}=19.1\right)$ and the surfactant polar parts provide good conditions for optimal MW heating where the electromagnetic wave is focused on the surface of each mini-reactor. However, the comparison between polyesterification in aqueous and bulk media at the same temperature, and using the DBSA as catalyst, reveals that the molecular weight of the obtained polyester is much higher in bulk with respect to water. This is probably due to the nature of the reaction media. Indeed, the presence of a large amount of water promotes competition between polyesterification and hydrolysis even though the presence of the surfactant would protect the mini-reactors.

\section{Acknowledgements}

The authors are thankful to ONIDOL Company for the financial support.

\section{References}

[1] Riffle, J.S., Freelin, R.G., Banthia, A.K. and McGrath, J.E. (1981) Interfacial Synthesis Part II: Phase-Transfer Catalyzed Synthesis of Polycarbonate/Polysiloxane Block Copolymers. Journal of Macromolecular Science, 15, 967-998. http://dx.doi.org/10.1080/00222338108056778

[2] Cassesa, E., Chao, D. and Henson, M. (1981) Cationic Surfactants in Interfacial Synthesis of Linear Aromatic Polyester. Journal of Macromelecular Science: Part A Chemistry, 15, 799-813. http://dx.doi.org/10.1080/00222338108056768

[3] Hanada, T. and Yanmitsu, O. (1971) Esterification of Model Peptides in Aqueous Solution. Chemical and Pharmaceutical Bulletin (Tokyo), 19, 1444-1449. http://dx.doi.org/10.1248/cpb.19.1444

[4] Allen, G. and Bevington, J. C. (1989) Comprehensive Polymer Science. Pergamon Press, Oxford.

[5] Saam, J.C. and Huebner, D.J. (1982) Condensation Polymerization of Oligomeric Polydimethylsiloxanols in Aqueous Emulsion. Journal of Polymer Science Polymer: Chemistry Edition, 20, 3351-3368. http://dx.doi.org/10.1002/pol.1982.170201209

[6] Saam, J.C. and Chou, Y.J. (1982) Method for Preparing Condensation Polymers by Emulsion Polymerization. US Patent 4355154.

[7] Chou, Y.J. and Saam, J.C. (1983) Method for Preparing Polyacetals and Polyketals by Emulsion Polymerization. US Patent 4374953.

[8] Baile, M., Chou, Y.J. and Saam, J.C. (1990) Direct Polyesterifcation in Aqueous Emulsion. Polymer Bulletin, 23, 251-257. http://dx.doi.org/10.1007/BF01032438

[9] Jong, L. and Saam, J.C. (1996) Polycondensation of Carboxylic Acid and Carinol in Heterogeneous Media. StepGrowth Polymers for High-Performance Materials, American Chemical Society Symposium Series, 624, 332-349. http://dx.doi.org/10.1021/bk-1996-0624.ch021

[10] Saam, J.C. (1998) Low-Temperature Polycondensation of Carboxylic Acids and Carbinols in Heterogeneous Media. Journal of Polymer Science Part A: Polymer Chemistry, 36, 341-356. http://dx.doi.org/10.1002/(SICI)1099-0518(19980130)36:2<341::AID-POLA17>3.0.CO;2-O

[11] Manabe, K., Sun, X.M. and Kobayashi, S. (2001) Dehydration Reactions in Water. Surfactant-Type Brønsted AcidCatalyzed Direct Esterification of Carboxylic Acids with Alcohols in an Emulsion. System, Journal of the American Chemical Society, 123, 10101-10102. http://dx.doi.org/10.1021/ja016338q

[12] Manabe, K., Limura, S., Sun, X.M. and Kobayashi, S. (2002) Dehydration Reactions in Water. Brønsted Acid-Surfactant-Combined Catalyst for Ester, Ether, Thioether, and Dithioacetal Formation in Water. Journal of the American Chemical Society, 124, 11971-11978. http://dx.doi.org/10.1021/ja026241j

[13] Barrère, M. and Landfester, K. (2003) Polyester Synthesis in Aqueous Miniemulsion. Polymer, 44, $2833-2841$. http://dx.doi.org/10.1016/S0032-3861(03)00151-4

[14] Tanaka, H. and Kurihashi, T. (2003) Synthesis of Polyesters by Emulsion Polycondensation Reaction in Water. Poly- 
mer, 35, 359-363.

[15] Takasu, A., Takemoto, A. and Hirabayashi, T. (2006) Polycondensation of Dicarboxylic Acids and Diols in Water Catalyzed by Surfactant-Combined Catalysts and Successive Chain Extension. Biomacromolecules, 7, 6-9. http://dx.doi.org/10.1021/bm050485p

[16] Manabe, K., Mori, Y., Wakabayashi, T., Nagayama, S. and Kobayashi, S. (2000) Organic Synthesis inside Particles in Water: Lewis Acid-Surfactant-Combined Catalysts for Organic Reactions in Water Using Colloidal Dispersions as Reaction Media. Journal of the American Chemical Society, 22, 7202-7207. http://dx.doi.org/10.1021/ja001420r

[17] Kobayashi, S., Uyama, H., Suda, S. and Namekawa, S. (1997) Dehydration Polymerization in Aqueous Medium Catalyzed by Lipase. Chemistry Letters, 26, 105. http://dx.doi.org/10.1246/cl.1997.105

[18] Namekawa, S., Uyama, H. and Kobayashi, S. (1998) Lipase-Catalyzed Ring-Opening Polymerization of Lactones in Water. Polymer Journal, 30, 269-271. http://dx.doi.org/10.1295/polymj.30.269

[19] Uyama, H., Inada, K. and Kobayashi, S. (1998) Enzymatic Polymerization of Dicarboxylic Acid and Glycol to Polyester in Solvent-Free System. Chemistry Letters, 27, 1285-1286. http://dx.doi.org/10.1246/cl.1998.1285

[20] Hoogenboom, R. (2007) Microwave-Assisted Polymer Synthesis: Recent Developments in a Rapidly Expanding Field of Research. Macromolecular Rapid Communications, 28, 368-386. http://dx.doi.org/10.1002/marc.200600749

[21] Gabriel, C., Gabriel, S., Grant, E.H., Halstead, B.S.J. and Mingos, D.M.P. (1998) Dielectric Parameters Relevant to Microwave Dielectric heating. Chemical Society Reviews, 27, 213-223. http://dx.doi.org/10.1039/a827213z

[22] Roussy, G. andPearce, J.A. (1995) Foundations and Industrial Applications of Microwave and Radio Frequency Fields: Physical and Chemical Processes. John Wiley \& Sons, Chichester.

[23] Silvestre, A.J.D., Gandini, A. and Neto, C.P. (2012) Synthesis of Aliphatic Suberin-Like Polyesters by Ecofriendly Catalytic Systems. High Performance Polymers, 24, 4-8. http://dx.doi.org/10.1177/0954008311431114

[24] Markus, A. and Landfester, K. (2002) Polyreaction in Miniemulsion. Progress in Polymer Science, 27, 689-757. http://dx.doi.org/10.1016/S0079-6700(01)00051-X 